\title{
Concerted Interactions between Multiple gp41 Trimers and the Target Cell Lipidome May Be Required for HIV-1 Entry
}

\author{
Biswajit Gorai, Anil Kumar Sahoo, Anand Srivastava, Narendra M. Dixit, and Prabal K. Maiti* \\ Cite This: J. Chem. Inf. Model. 2021, 61, 444-454 \\ Read Online
}

ABSTRACT: The HIV-1 envelope glycoprotein gp41 mediates the fusion between viral and host cell membranes leading to virus entry and target cell infection. Despite years of research, important aspects of this process such as the number of gp41 trimers involved and how they orchestrate the rearrangement of the lipids in the apposed membranes along the fusion pathway remain obscure. To elucidate these molecular underpinnings, we performed coarse-grained molecular dynamics simulations of HIV-1 virions pinned to the CD4 T cell membrane by different numbers of gp41 trimers. We built realistic cell and viral membranes by mimicking their respective lipid compositions. We found that a single gp41 was inadequate for mediating fusion. Lipid mixing between membranes, indicating the onset of fusion, was efficient when three or more gp41 trimers pinned the membranes. The gp41 trimers interacted strongly with many different lipids in the host cell membrane, triggering lipid configurational rearrangements, exchange, and mixing. Simpler

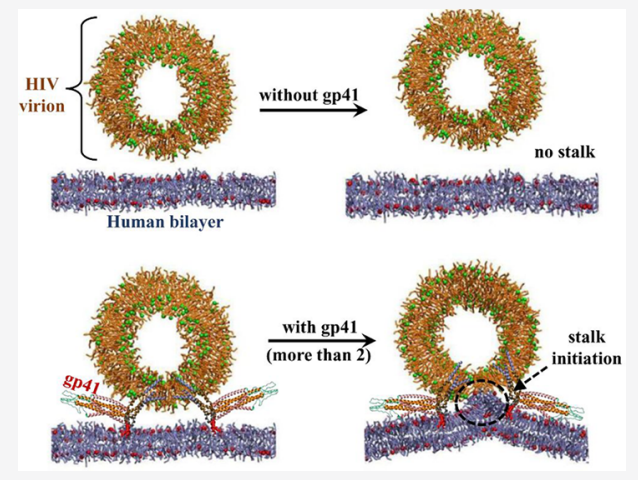
membranes, comprising fewer lipid types, displayed strong resistance to fusion, revealing the crucial role of the lipidomes in HIV-1 entry. Performing simulations at different temperatures, we estimated the free energy barrier to lipid mixing, and hence membrane stalk formation, with three and four tethering gp41 trimers to be $\sim 6.2 \mathrm{kcal} / \mathrm{mol}$, a $>4$-fold reduction over estimates without gp41. Together, these findings present molecular-level, quantitative insights into the early stages of gp41-mediated HIV-1 entry. Preventing the requisite gp41 molecules from tethering the membranes or altering membrane lipid compositions may be potential intervention strategies.

\section{INTRODUCTION}

The entry of HIV-1 into its target T helper (CD4 T) cells is mediated by its surface envelope protein, Env, a homotrimer of noncovalently linked heterodimers of glycoproteins gp120 and gp41. ${ }^{1-4}$ Entry begins with gp120 binding to the cell surface receptor $\mathrm{CD} 4$, following which conformational changes in gp120 enable its binding to one of the coreceptors CCR5 or CXCR4. Further conformational changes ensue, resulting in the exposure of the $\mathrm{N}$-terminal fusogenic domain of gp41, also called the fusion peptide (FP; see Figure S1), its insertion into the target cell membrane, and membrane fusion leading to virus entry. ${ }^{2}$ Blocking virus entry is an important intervention strategy. Drugs or dendrimers that block CD4/CCR5 binding to gp $120^{5-7}$ or conformational changes in gp41 facilitating entry ${ }^{8}$ are approved for clinical use. Vaccination strategies also aim to administer or elicit broadly neutralizing antibodies that target Env and prevent entry. ${ }^{9}$ The success of these strategies relies on a detailed, quantitative understanding of the molecular requirements of the entry process, which, despite years of research, is still lacking. For instance, how many Env trimers are required for entry is yet to be established. ${ }^{8}$

While attention has been focused in previous studies on the proteins involved in the entry process, ${ }^{4,10-12}$ much less is known of the roles played by the lipids involved despite intermembrane lipid mixing being central to membrane fusion.
Lipid mixing is an important barrier to fusion given that the lipidomes of the HIV-1 and target cell membranes are vastly different. ${ }^{13}$ The proteins and lipids must thus work in tandem to orchestrate virus entry.

Here, to elucidate the roles of both the lipids and the associated proteins in HIV-1 entry, we performed detailed, long-time scale $(\sim 10 \mu \mathrm{s})$, coarse-grained molecular dynamics (CGMD) simulations of the viral and host cell membranes held in close apposition by gp41 trimeric tethers. We faithfully mimicked the known compositions of the viral and host lipidomes, leading to the creation of complex, multilipid-type membranes. By varying the number of gp41 tethers, we estimated the protein requirements for lipid mixing and their energetic contributions. At the same time, we unraveled the detailed configurational changes of the lipids culminating in mixing and eventually membrane fusion. Specifically, we answered the following questions: (i) how many gp41 trimers

Received: November 6, 2020

Published: December 29, 2020 

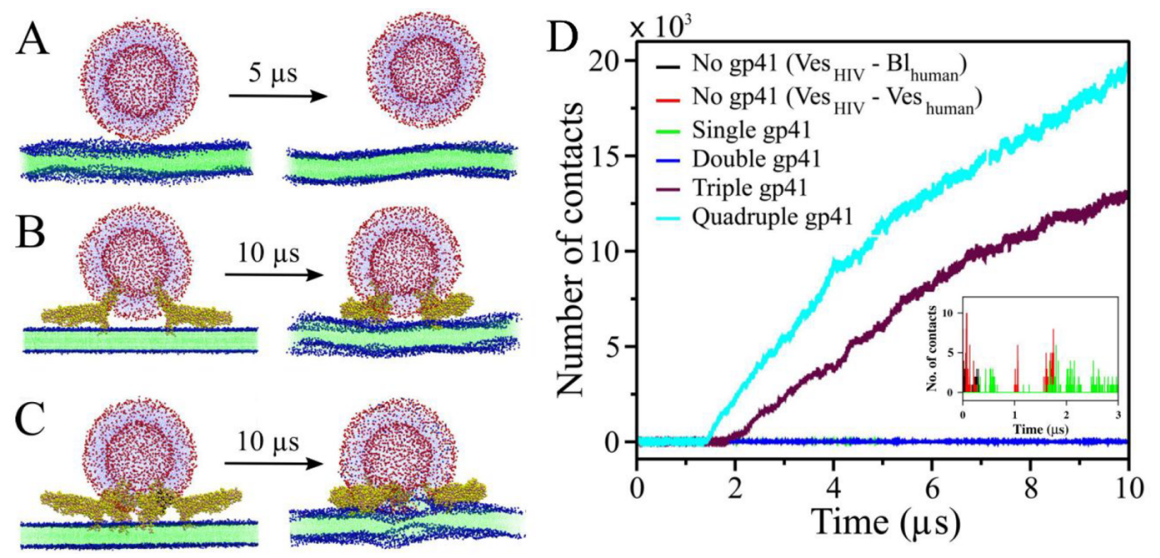

Figure 1. Stoichiometry of gp41 for membrane fusion. The instantaneous snapshots of the initial (left) and final (right) configurations of the system for fusion of the human bilayer-HIV-1 vesicle in the presence of (A) no, (B) double, and (C) quadruple gp41 trimeric units are depicted. (D) The number of contacts among intermembrane lipid beads is shown for the simulated systems at $300 \mathrm{~K}$ with different gp 41 trimeric units $(0-$ 4), which form bridges between the human bilayer and the HIV-1 vesicle membrane models. Note that black, red, and green lines are near zero and shrouded by blue, brown, and cyan lines. A plot (up to $3 \mu \mathrm{s}$ ) in the inset shows the lipid contacts for the systems with less than two gp41 trimers. The exchange of lipids between the human bilayer and the HIV-1 vesicle is observed only in the presence of three and four gp 41 trimers. The lipid head groups and tails of the human bilayer are shown in vdW (blue) and line (green) representations, respectively. The lipid head groups and tails of the HIV-1 vesicle are shown in vdW (red) and line (light blue) representations, respectively. The gp41 trimeric unit is shown in vdW representation with a yellow color. Water and ions are not shown here for clarity.

are required for membrane fusion, (ii) how do they orchestrate lipid mixing, (iii) what is the associated free energy barrier, and (iv) how important are the lipid compositions to membrane fusion.

\section{RESULTS}

More than Two gp41 trimeric Units Are Necessary for Fusion. Previous studies, using experiments and simulations, have demonstrated the possibility of membrane fusion without the involvement of any fusogenic peptides or proteins. ${ }^{14-16} \mathrm{We}$ therefore first performed simulations containing the HIV-1 and human $\mathrm{T}$ cell membrane models in the absence of gp41. The cell membranes of HIV-1 and host cell are heterogeneous and mainly constructed by cholesterol, PC, PE, PS, and SM lipid types. The composition of the lipids varies substantially within the leaflets and within local domains in each leaflet. ${ }^{17}$ The membranes differed in their overall lipid compositions as well as in the compositions of their inner and outer leaflets (Methods). The lipid compositions of the membranes used in our study are comparable (within $\pm 10 \%$ tolerable range) to those in other recent studies. ${ }^{13}$ Cholesterol-rich domains or lipid rafts in the host cell are known to facilitate fusion. ${ }^{18} \mathrm{We}$ have therefore modeled the host cell membrane with a higher percentage of cholesterol (37.2\%). We built HIV-1 as a vesicle of $20 \mathrm{~nm}$ diameter and held it initially in close proximity to a flat $40 \times 40 \mathrm{~nm}^{2}$ cell membrane (Figure 1A), representative of the virus-host cell membrane interactions considering the cell diameter is nearly 100 -fold the virion diameter. Rendering the cell membrane as a vesicle of $20 \mathrm{~nm}$ diameter did not alter our findings (Figure S2). We found that the membranes drifted away from each other in our $5 \mu \mathrm{s}$ simulations (Figures $1 \mathrm{~A}$ and S2). A previous study with vesicles composed of 1-2 lipid types found them repelling during CGMD simulation. ${ }^{19}$ The electrostatic repulsion due to the similarly charged lipid head groups and the hydration force seems to drive the membranes apart. ${ }^{20}$ Within the duration of our simulations, thus, the membranes were resistant to spontaneous fusion. We, therefore, examined next whether gp41 trimers could overcome this resistance.

We now initialized our simulations with the membranes apposed as above but tethered to each other by different numbers of gp41 trimers (Figures 1B,C and S3). The gp41 trimers were placed symmetrically around a focal region. We found that with one and two gp41 trimers, the membranes remained apposed but formed no intermembrane lipid contacts in the $10 \mu \mathrm{s}$ simulations (Figure 1D). Extending the simulation with two gp41 trimers from 10 to $20 \mu \mathrm{s}$ did not change the scenario (Figure S4). With three gp41 trimers, the number of contacts increased rapidly starting at $1.8 \mu \mathrm{s}$, indicating the onset of lipid mixing. With four trimers, mixing began earlier, at $1.4 \mu \mathrm{s}$, and proceeded faster. These simulations demonstrate that, for the initial gp41 configurations chosen, at least three trimers were necessary to initiate fusion. This finding is consistent with recent experiments showing that most HIV-1 strains require two to three trimers for entry. ${ }^{10}$ Further, fusion was expedited with more trimers involved.

gp41 Trimers Act Cooperatively to Initiate Membrane Fusion. The multiple gp41 trimers involved in fusion initiation could act independently or cooperatively. To ascertain this, we tracked the movements of the trimers throughout the simulations. Specifically, we estimated the distances between the center-of-mass (COM) of every pair of gp41 trimers, considering their fusion peptides and transmembrane domains. We numbered the trimeric units, U1, U2, etc. We found that the gp41 units were mobile throughout the $10 \mu$ s long simulation and had a tendency to cluster transiently (Figure 2). For the system with three gp41 trimers, U1 and U3, which were initially separated by $\sim 9 \mathrm{~nm}$, approached each other, reached a minimum separation of $\sim 3.5 \mathrm{~nm}$ at $\sim 2 \mu \mathrm{s}$, and then moved away (Figure 2A,C). Interestingly, the time at minimum separation corresponded to the time when intermembrane contacts began to increase rapidly (Figure 1D). With the four gp41 trimer system too, U1 and U2 achieved a minimum separation similar to that above and at a 

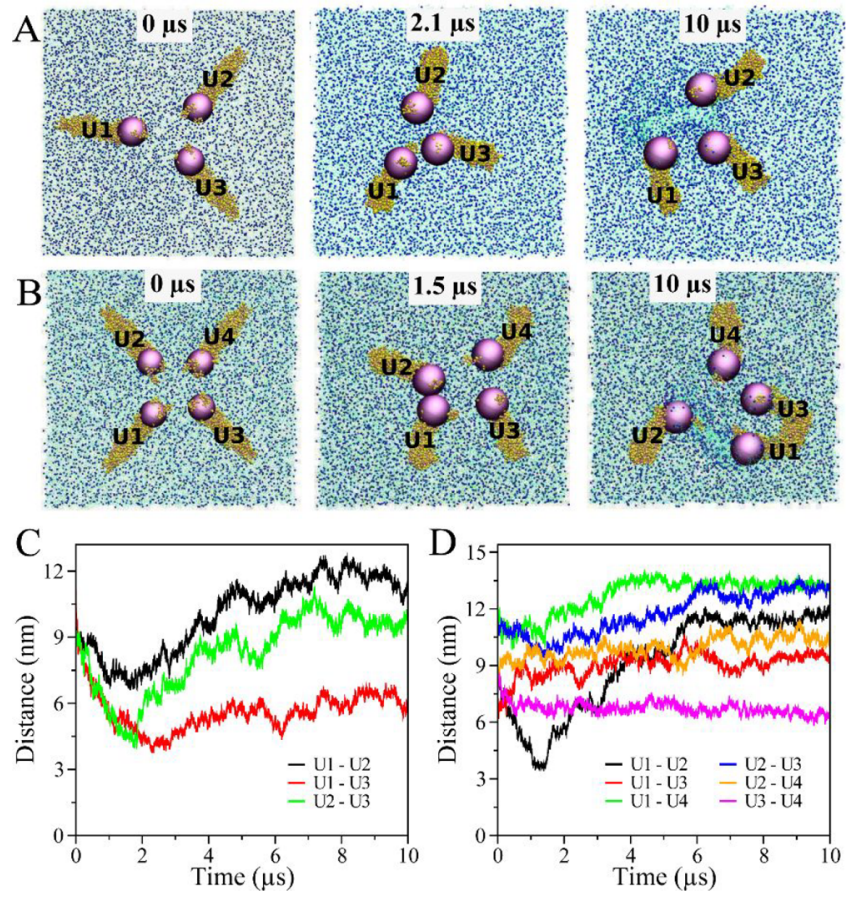

Figure 2. Cooperative action of gp41 trimer units (U) to initiate lipid mixing and membrane fusion. The snapshots at the initial, onset of stalk formation, and final simulation time instances for systems with (A) triple (U1-U3) and (B) quadruple (U1-U4) gp41 units are depicted. The gp41 trimeric units and the center-of-mass (CoM) of the FP+TMD domains of each gp41 trimer are represented by yellow beads and violet spheres, respectively. The hydrophobic tails and the polar heads of the human bilayer lipids are represented by green lines and blue vdW spheres, respectively. The simulation time is inscribed on the top-middle of each figure. The HIV-1 vesicle, water beads, and ions are not shown in the snapshots for clarity. For each pair of gp41 trimeric units, the distance (in the $X-Y$ plane) between the CoMs of the FP+TMD domains as a function of the simulation time is shown for systems with (C) triple and (D) quadruple gp41 units. The gp41 trimeric units seem mobile in nature and approach each other (with the minimum possible separation of $\sim 3.5 \mathrm{~nm}$ ) to initiate the stalk formation, which facilitates intermembrane lipid mixing. After the stalk formation process completes, the separations between gp41 trimer units escalate to further proliferate the stalk elongation.

time, $\sim 1.5 \mu \mathrm{s}$, that corresponded well with the rise of intermembrane contacts (Figures 2D and 1D). The other pairs did not come this close. It appears thus that when COM of two gp41 trimers approach each other within a separation of $\sim 3.5 \mathrm{~nm}$, they can trigger the formation of lipid contacts and initiate membrane fusion. The additional trimers perhaps serve to stabilize the membranes when lipid mixing is ongoing. To check the accumulation of trimer units in a more visually informative way, we have calculated the contact map between the gp41 trimer units in triplet and quadruplet systems (Figure 3 ). Briefly, the minimum distance between every residue of each monomer $\left(\mathrm{m}_{1}\right.$ to $\left.\mathrm{m}_{3}\right)$ of the trimer units of the stalk initiation conformers (2.1 and $1.5 \mu \mathrm{s}$ for triplet and quadruplet systems, respectively) are calculated and plotted in a heat map. Clearly, the FP and TMD domains are closest and the IL domains farthest among the interacting monomer units. It is possible that in our simulations with two gp41 trimers, because the trimers were placed diametrically opposite each other, they never approached such close separations within our simulation time. Env spikes of a mature HIV-1 virion have been observed to form small clusters, ${ }^{21}$ possibly to drive fusion. To the best of our knowledge, we report for the first time the minimum distance of approach between two gp41 trimeric units that is essential to initiate intermembrane lipid contact and hence fusion. This observation may have implications for vaccines aimed at neutralizing HIV-1 virions. ${ }^{9}$ We turn next to the role of lipids.

Viral and Cell Lipidomes Promote Membrane Fusion. Cell membranes are composed of several lipid types. Further, the lipid compositions of the inner and outer leaflets of membranes can be quite different. Most computational and experimental studies consider simple membrane models composed of only one or at best a few lipid types. ${ }^{22}$ To examine whether such simple models capture the HIV-1 fusion process, we performed simulations with the HIV-1 vesicle and the apposed cell membrane both composed of POPC (80\%) and POPS (20\%) CG lipids, tethered by four gp41 trimers (Figure S5). We found no intermembrane contact formation within the $10 \mu \mathrm{s}$ long simulations performed at the regular temperature $(300 \mathrm{~K})$ or an elevated temperature $(400 \mathrm{~K})$. As we showed above, however, membrane models composed of near biological lipid compositions formed lipid contacts and mixed (Figure 1D). The lipidomes of the virus and cell membranes are thus important to membrane fusion; they appear to accelerate fusion. We examined next how the lipidomes participated in the fusion process.

Differential Splaying of Lipid Types May Promote Fusion. Membrane fusion involves changes in membrane lipid configurations as well as mixing and homogenization of lipid compositions. At the local level, configurational changes may manifest as tilt and splay. We examined both and compared them between lipids near the focal, contact region, within a 20 $\AA$ Aistance, and those far away. Initially, the lipids exhibited no tilting and were aligned along the bilayer normal (Figure S6). At the onset of lipid mixing, substantial tilt away from the normal was seen, in keeping with the bending membrane (Figure S6). Further, different lipid types showed different extents of tail splay in the process, quantified as the probability distribution of the splay angle between the aliphatic tails (Figure 4). In the absence of fusion proteins, splaying of lipid tails facilitates membrane fusion. ${ }^{23}$ Away from the contact region, the lipids were unstressed and exhibited splay within a narrow range, $35^{\circ}-42^{\circ}$. In the contact region, the splay varied far more, $20^{\circ}-60^{\circ}$. DOPS, with one double bond in both the aliphatic tails, showed the highest splay. The splay angle of a few DOPS and POPS molecules, which are found mostly in the inner leaflets, exceeded $60^{\circ}$, consistent with the greater splay of inner leaflet lipids observed with the DMPC/DOPE membrane system. ${ }^{24}$ These observations suggest that some lipids splay more than others and absorb more of the stress associated with membrane bending. Other lipids that are less flexible, namely DPPC and DPPE, can get away with much less splay and perhaps even contribute to membrane integrity. In a model membrane lacking this lipidome diversity, the lipids may either all splay, leading to poorer integrity, or not splay at all, preventing fusion. The lipidome diversity may thus serve as an optimum in this integrity-flexibility trade-off.

Lipid Transfer between Membranes Is Asymmetric and Asynchronous. To quantify lipid mixing between the membranes, we tracked the number of lipids transferred from the cell membrane to the HIV-1 vesicle $(\mathrm{C} \rightarrow \mathrm{H})$ and the same from the HIV-1 vesicle to the cell membrane $(\mathrm{H} \rightarrow \mathrm{C})$ for each lipid type. For each lipid type, $\mathrm{C} \rightarrow \mathrm{H}$ transfer was 

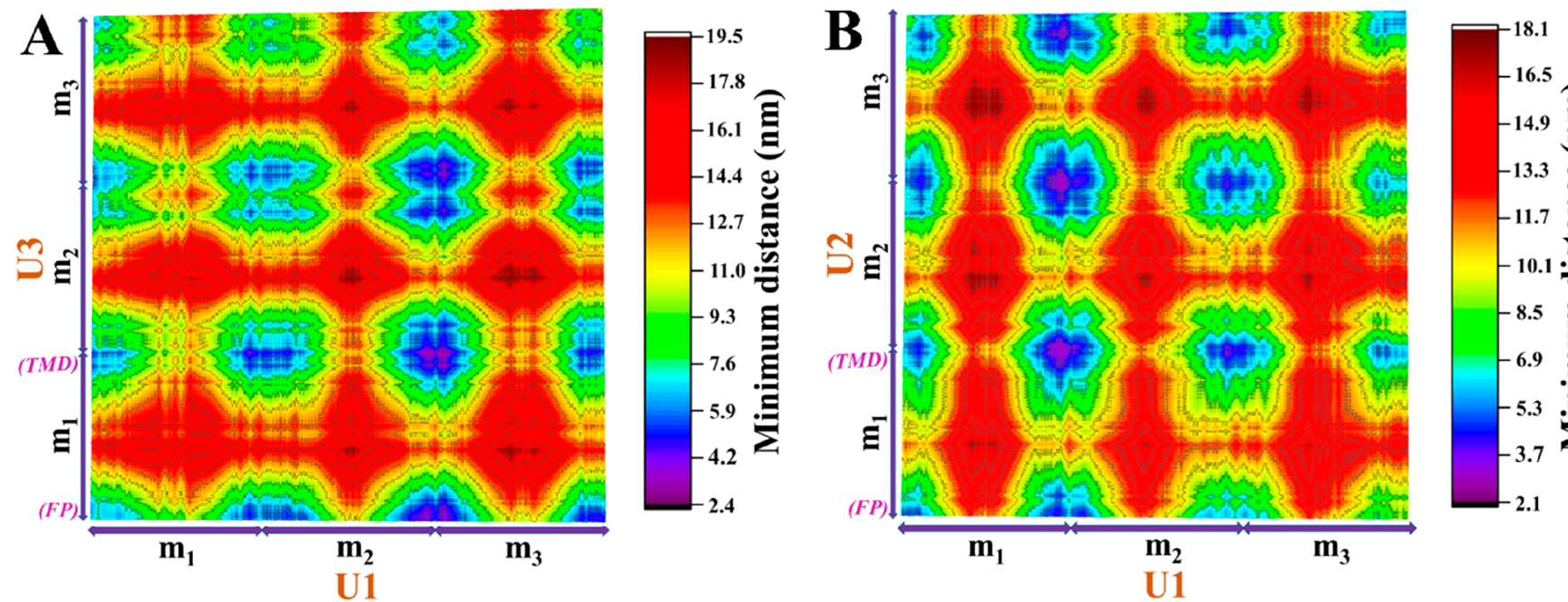

Figure 3. HIV-1 gp41 trimer contact map. (A) The contact map estimated based on the minimum distance between the residues of trimer units (U1 and U3) in a triplet system at the onset of stalk formation (snapshot at $2.1 \mu \mathrm{s}$ ). (B) The contact map estimated based on the minimum distance between the residues of trimer units ( $\mathrm{U} 1$ and $\mathrm{U} 2)$ in a quadruplet system at the onset of stalk formation (snapshot at $1.5 \mu \mathrm{s}$ ). The monomers of each gp41 trimer are represented by $\mathrm{m}_{1}, \mathrm{~m}_{2}$, and $\mathrm{m}_{3}$. The $\mathrm{N}$ - and C-terminals of the monomers represent the FP and TMD domains, respectively. The variation in the minimum distance between the residues of the monomers of the gp 41 can be estimated from the color scales on the right side of each panel.
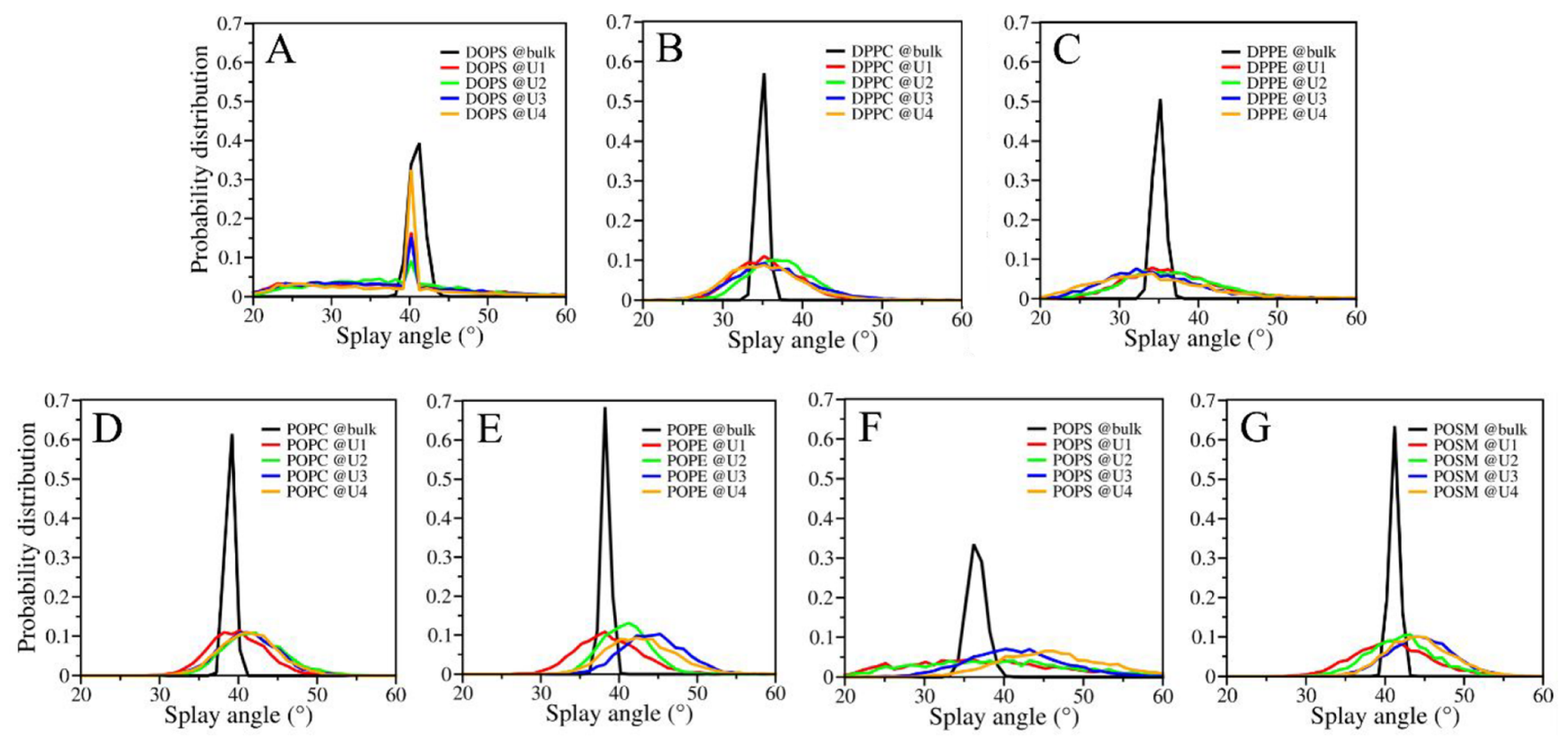

Figure 4. Splay angle probability distribution. The normalized probability distribution of the splay angle between the two tails of (A) DOPS, (B) DPPC, (C) DPPE, (D) POPC, (E) POPE, (F) POPS, and (G) POSM lipids at bulk (black line) and lipids around gp41 (20 Å of center-of-mass of $\mathrm{FP}+\mathrm{TMD}$ domain) of each trimeric unit (U1-red line, U2-green line, U3-blue line, and U4-orange line) is shown.

observed immediately after the lipid contact initiation time $(\sim 1.4 \mu \mathrm{s})$, whereas $\mathrm{H} \rightarrow \mathrm{C}$ transfer occurred much later, $\sim 4 \mu \mathrm{s}$ (Figure 5). Also, the $\mathrm{C} \rightarrow \mathrm{H}$ transfers (in $10 \mu \mathrm{s}$ ) were fewer than $\mathrm{H} \rightarrow \mathrm{C}$ transfers. The cell membrane underwent significant morphological changes during the process, a transient dimple formed leading to stalk initiation (Figure S7 and Movie S1), whereas the HIV-1 membrane maintained its spherical geometry. The HIV-1 membrane contains more cholesterol $(\sim 45 \%)$ than the cell membrane ( $37 \%)$, which provides it more order and rigidity, possibly explaining the fewer successful lipid transfers to the vesicle than from it. In descending order of numbers, $\mathrm{C} \rightarrow \mathrm{H}$ transfers followed: cholesterol $>$ POPC $>$ POSM $\approx \mathrm{DPPC}>\mathrm{POPE} \approx \mathrm{DPPE}$; whereas $\mathrm{H} \rightarrow \mathrm{C}$ transfers followed: cholesterol $>\mathrm{POPE} \approx$ $\mathrm{POSM}>\mathrm{POPC} \approx \mathrm{POPS}$. We have calculated the lateral diffusion coefficient of the each coarse-grained lipid using the "gmx msd" module of GROMACS in the human bilayer (Table S1) before and after stalk formation. The values typically lie within the range $10^{-8}-10^{-7} \mathrm{~cm}^{2} \mathrm{~s}^{-1}$, which are in agreement with the reported values. ${ }^{28-30}$ The diffusion coefficient of each lipid marginally increased after stalk formation, which further supports the higher lipid transfer after stalk initiation. 

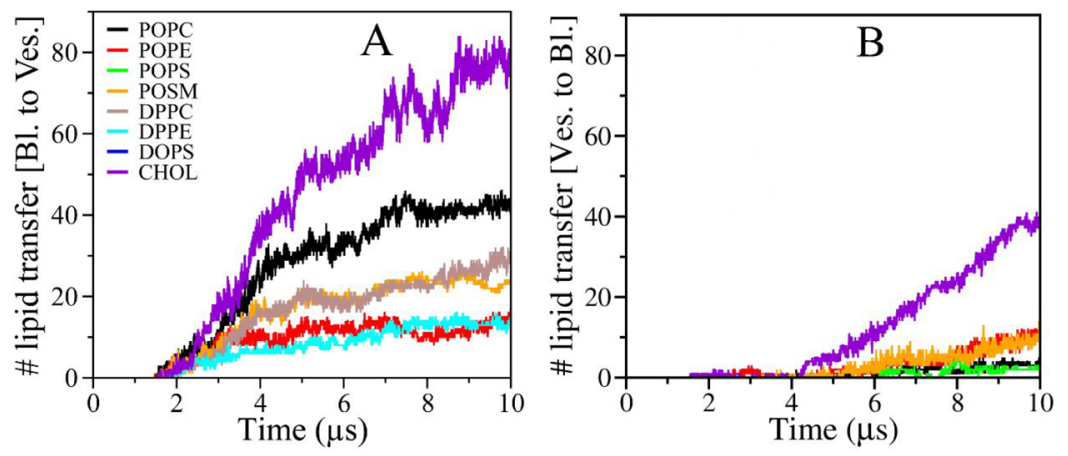

Figure 5. Kinetics of intermembrane lipid transfer. For each lipid type, the number of lipids transferred from the human membrane bilayer to the HIV-1 vesicle (A) and from the HIV-1 vesicle to the human membrane bilayer (B) is plotted as a function of the simulation time. The human membrane contains every lipid type except POPS, while the HIV-1 vesicle contains only POPC, POPE, POPS, POSM, and cholesterol.
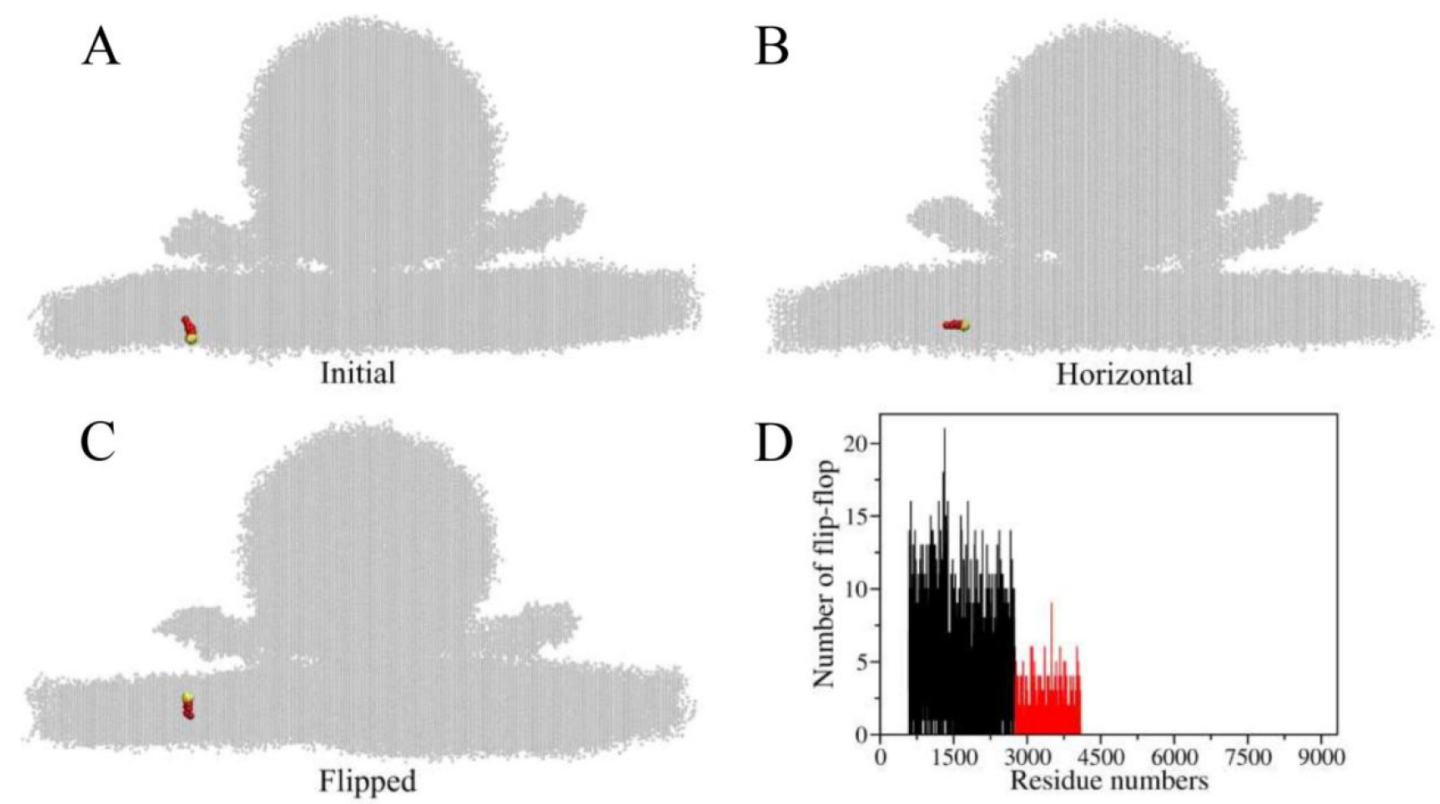

Figure 6. Flip-flop of lipids. Snapshots displaying the major events during the flip-flop pathway of cholesterol: (A) initial stage: cholesterol in the lower leaflet, (B) intermediate stage: horizontal alignment of cholesterol, and (C) final stage: cholesterol moves to the upper leaflet. The hydroxyl headgroup and nonpolar fatty acid tail of cholesterol are represented by yellow and red vdW spheres, respectively. (D) The number of times each lipid flipped between the leaflets, before stalk formation, is shown. We noticed that only cholesterols (residue number 583-4084) underwent translocation within the leaflets, and the rate of translocation between leaflets is higher for cholesterols of human bilayer (black: residue number 583-2749) than cholesterols of the HIV-1 vesicle (red: residue number 2750-4084).

As cholesterol has the ability to flip-flop between the inner and outer leaflets (Figure 6) and induce negative curvature to the membrane, ${ }^{25}$ it shows the highest tendency to translocate between the membranes in the focal region. In our simulations, other lipid types did not flip even once within $10 \mu$ s (Figure 6); transfers were thus predominantly of the lipids in the outer leaflets. For all lipid types except cholesterol, the transfers saturated after $8 \mu \mathrm{s}$, indicative of the achievement of new stable compositions. (Figure 5). Intriguingly, from the simulation result we are able to explain for the first time the higher rigidity of the viral membrane than that of the host membrane. Our observation is consistent with the experiments performed from fluorescent spectroscopy, inferring that the host cell membranes are notably less stable than the virus envelope. ${ }^{26,27}$

gp41 Domains Trigger Local Alterations in Lipid Compositions and Morphology. Given that no lipid mixing occurred in the absence of gp41 tethers, we examined the interactions between gp41 and the cell membrane lipids, which could potentially have facilitated the mixing. We noticed a marginal elevation in the concentrations of POPC, POPE, POSM, and cholesterol in the focal region (Figure S8). The interactions of functional domains of gp41 with lipid membranes have been studied using different biochemical and biophysical techniques. ${ }^{31}$ However, their preferences for lipid types and their roles in membrane fusion remain unclear. We calculated the two-dimensional radial distribution functions (RDF) of the various membrane components around each gp41 functional domain and used the heights of the first peaks in the RDFs to infer the lipid association propensities. The resulting preferences are summarized in Figure 7 . The transmembrane domain (TMD) showed the highest preference for cholesterol, followed by the negatively charged POPS and the zwitterionic POPE lipids. The fusion peptide (FP) too showed the highest preference for cholesterol followed by zwitterionic lipids. It, however, showed minimal affinity for the negatively charged lipids. The membrane-proximal external 


\begin{tabular}{|c|c|c|c|c|c|c|c|c|}
\hline \multirow{2}{*}{ gp41 domains } & \multicolumn{7}{|c|}{ Membrane components (lipids + sterol) } \\
\hline & POPC & POPE & POPS & DPPC & DPPE & DOPS & POSM & CHOL \\
\hline TMD & & & & & & & & \\
\hline FP & & & & & & & & \\
\hline CRAC & & & & & & & & \\
\hline MPER & & & & & & & & \\
\hline FPPR & & & & & & & & \\
\hline CHR & & & & & & & & \\
\hline IL & & & & & & & & \\
\hline NHR & & & & & & & & \\
\hline
\end{tabular}

Figure 7. Summary of the interactions of various gp41 functional domains with the different lipid types. The occupancy of each lipid type around the functional domains of gp41 is depicted in the tabular form. The likeliness of each gp41 functional domain (when present in the six-helix bundled conformer) for the lipids varies from strong to weak, which is represented by the dark blue-to-white color gradient. TMD and FP domains of gp41, initially placed in the HIV-1 and human membranes, respectively, show the maximal fondness for the lipids, which is followed by CRAC, MPER, FPPR, CHR, and IL. Note that there is no interaction between NHR and the lipids, as NHR is enclosed by the CHR helices in the six-helix bundle (6HB) postfusion conformer of the gp41 trimer (Figure S1A). The relative occupancy of each lipid type around the functional gp41 domains varies from strong to weak, which are represented in the dark brown-to-white color gradient. As the anionic DOPS lipid is only present in the inner leaflet of the human bilayer, it does not interact with any functional domain of gp41. All other lipid types are found to interact with at least one of the functional domains of gp41.

region (MPER) too showed the highest preference for cholesterol, followed by zwitterionic and anionic lipids. The later is argued to be due to MPER's cholesterol recognition amino acid consensus (CRAC) motif found at the C-terminal end. $^{32}$ RDFs for the CRAC motif also showed that only cholesterol was present near it. Note that MPER is considered as the functional counterpart of FP and plays a crucial role in bridging HIV-1 and host membranes. ${ }^{31}$ The fusion peptide proximal region (FPPR) domain was surrounded by zwitterionic lipids more than anionic lipids or cholesterol. The C-terminal heptad repeat (CHR) preferred the negatively charged POPS the most, followed by the unsaturated zwitterionic lipids. On the other hand, the immunodominant loop (IL) showed higher affinities for zwitterionic lipids followed by cholesterol.

A recent experimental study has argued that cholesterol-rich domains facilitate gp41-mediated membrane fusion. ${ }^{18}$ Our simulations showed the preferred localization of cholesterol around FP, CRAC, and MPER domains of gp41. This localization, given the hydrophobic nature of cholesterol, would reduce the unfavorable interactions between the apposed membranes near the gp41 tethers. When a sufficient number of gp41 trimers accumulated, the opposing interactions were sufficiently reduced to allow lipid transfers and mixing. Our study thus reveals the crucial role of FP, CRAC, and MPER domains in membrane stalk formation. They cause local redistributions of the lipid types, enriching cholesterol, thereby mitigating membrane repulsions and facilitating fusion.

The significance of the IL domain during fusion is not well understood. We find from the simulations that IL anchors into the cell membrane and induces membrane bending. Other domains seem to play an insignificant role in gp41-mediated membrane fusion. The location of the L173 (N-terminal residue of TMD) and R185 (arginine at the center of TMD) from the center of mass of the local membrane (lipids within 2 $\mathrm{nm}$ of TMD) was estimated (Figure 8). We observed that

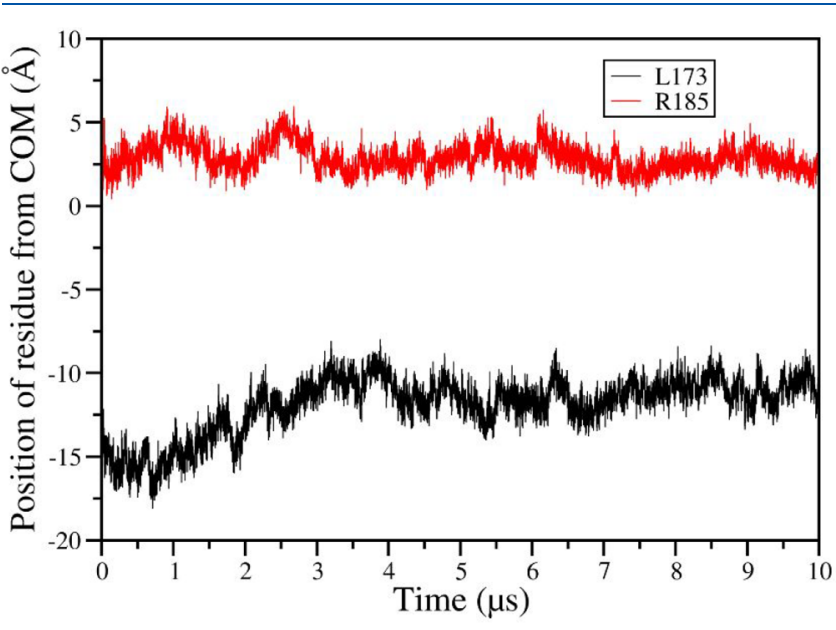

Figure 8. Position of TMD residues in the HIV-1 membrane. The variation in the location of the L173 (residue lying on the edge of the upper leaflet of the HIV-1 membrane) and R185 (residue situated at the center of the viral membrane) from the COM of the lipids (within $2 \mathrm{~nm}$ of TMD) w.r.t. the simulation time is shown in black and red lines, respectively.

$\mathrm{R} 185(2.88 \pm 0.83 \AA)$ lay near the center of the membrane throughout the simulation. The L145 $(11.92 \pm 1.72 \AA)$ is initially $\sim 15 \AA$ away from the COM of the membrane (i.e., on the edge of the upper leaflet of the HIV-1 membrane). However, the distance gradually reduced to $\sim 10 \AA$ after $3 \mu \mathrm{s}$, which probably is due to the thinning of the HIV-1 membrane during the stalk initiation time frame.

We have calculated the change in tilt angle of FP, TMD, and $\mathrm{FP}+\mathrm{TMD}$ domains of gp41 during the simulation time (Figure S9). While estimating the trimer tilt, the angle between the vector of $\mathrm{FP} / \mathrm{TMD} / \mathrm{FP}+\mathrm{TMD}$ terminal beads (COM of first beads and last beads) and bilayer normal was calculated. The tilt angles of the FP's of the system with four gp41 trimer units involved in forming clusters (U1 and U2) partially decrease until stalk initiation and later become stabilized. The tilt of TMDs of U1 and U2 gradually increases until stalk formation and thereafter becomes stabilized. However, the tilt angle of terminals of FP+TMD w.r.t. the cell membrane normal initially decreases and later increases after stalk formation.

gp41 Reduces the Activation Barrier for Membrane Stalk Formation. The free-energy landscape for HIV-1-cell membrane fusion is complex (Figure 9A), as it encompasses several intermediate processes including the formation of the membrane stalk, the hemifusion diaphragm, and the final fusion pore. ${ }^{33}$ It is known from various studies that the freeenergy barrier for the initial stalk formation, in the absence of proteins, is quite high $(\sim 40-220 \mathrm{kT}){ }^{34}$ mainly due to the electrostatic repulsion between the polar head groups and the hydration repulsion. Our simulations showed that gp41 trimers facilitate lipid mixing, leading to stalk formation. To quantify the extent to which gp41 trimers reduce the free-energy barrier $\left(\Delta G_{S}\right)$, we performed simulations with three and four gp41 trimers tethering the membranes (Figure 1C) at different temperatures in the range $300-400 \mathrm{~K}$. We found that the time of onset of lipid contact formation, or the stalk initiation time, $\tau$, decreased with the temperature, $T$ (Table S2). This implied that stalk formation is an activated process, as depicted in 


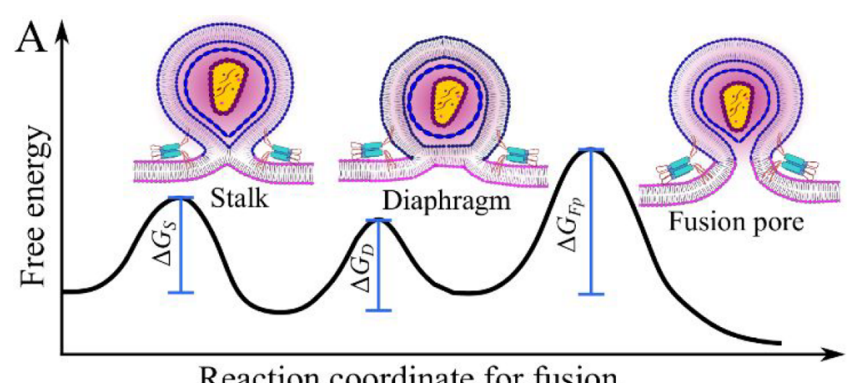

Reaction coordinate for fusion

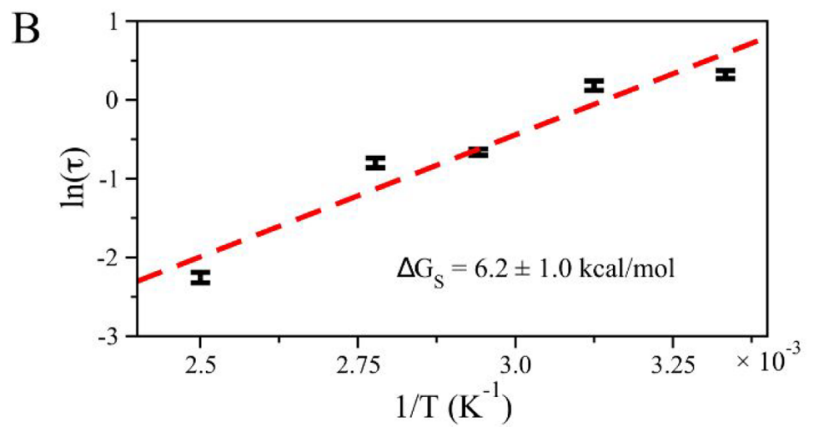

Figure 9. Activation barrier for the gp41-mediated stalk formation. (A) Schematic of the free-energy landscape for the human-HIV-1 cell membrane fusion, comprising of the activation barriers for the initial stalk formation $\left(\Delta G_{S}\right)$, the intermediate stalk elongation or hemifusion diaphragm $\left(\Delta G_{D}\right)$, and the final fusion pore formation $\left(\Delta G_{F p}\right)$, is shown. Each of the three energy-driven processes along the fusion pathway is inscribed in the schematic. The human cell is shown as a membrane bilayer with magenta color lipid heads, whereas the HIV-1 virion, enclosing the capsid, is depicted as a vesicle with blue color lipid heads. The gp41 trimeric unit is shown in cyan color. (B) The natural logarithm of the stalk initiation time $(\tau)$ is plotted as a function of the inverse temperature. The five data points (from right to left) correspond to temperatures $300,320,340,360$, and $400 \mathrm{~K}$, respectively. The error bar is the standard deviation of the mean $\tau$ estimated from two independent simulations at each temperature. $\Delta G_{S}(=6.2 \pm 1.0 \mathrm{kcal} / \mathrm{mol})$ is obtained by linear fit (dashed line) of the data points to the Arrhenius formula, $\ln \tau=\ln \tau_{0}+\Delta G_{S} / k_{\mathrm{B}} T$.

Figure 9A. We used the temperature dependence of $\tau$ to estimate $\Delta G_{S}$ using the Arrhenius equation, $\tau=\tau_{0} \exp \left(\Delta G_{S}\right)$ $\left.k_{\mathrm{B}} T\right)$, where $\tau_{0}$ is the intrinsic time constant, and $k_{\mathrm{B}}$ is Boltzmann's constant. We found that $\Delta G_{S}$ was $6.20 \pm 1.7 \mathrm{kcal} /$ $\mathrm{mol}$ and $6.16 \pm 1.0 \mathrm{kcal} / \mathrm{mol}$ with three and four gp41 trimers, respectively (Figure 9B). This is a reduction of $>4$-fold compared to the barrier without fusion proteins, ${ }^{34}$ indicating the critical role played by gp41 in facilitating HIV-1 entry. The lipid mixing is expedited with increasing gp 41 trimers ( 3 to 4 ) at different temperatures (Table S3). It follows that $\Delta G_{S}$ obtained with three and four gp41 trimers does not change significantly, probably because we are working with a model system with smaller than the natural dimensions of host and HIV-1 membranes. The engagement with the host cell is a highly regulated process (through receptors CD4 and CCR5/ CXCR4), and localization of several receptors at the site of fusion may also be energetically unfavorable, possibly also underlying the similar $\Delta G_{S}$ with three or four gp41 trimers. We further anticipate that the energy barrier obtained in the current study may be influenced by several factors. Specifically it depends on the simulation run-time. Also the cytoplasmic (CP) domain of gp41, which is known to be involved in fusion, is absent in our gp41 model.

\section{DISCUSSION}

Previous studies have inferred that the number of fusogenic proteins required for HIV-1 entry varies from 1-8, with most HIV-1 strains requiring $2-3 .^{10,35}$ In accordance, our study shows that at least three gp41 trimers were necessary for membrane fusion initiation. Further, the fusion proteininduced reduction of the free energy barrier to membrane fusion we estimated is in keeping with recent experimental and theoretical studies. ${ }^{10,36,37}$ We find that the barrier reduces with the number of gp41 trimers involved and that with four gp 41 trimers, the barrier is reduced $>4$-fold over that in the absence of the trimers. Antibodies that target gp41, especially the MPER region, have been identified and can neutralize a wide variety of HIV-1 strains. ${ }^{38,39}$

Our study, like the previous ones, ${ }^{10}$ provides a quantitative guideline for the use of such antibodies. The clustering of the Env proteins observed near the virus-cell contact region ${ }^{4}$ implies that nearly all of the Env trimers may be involved in tethering the membranes. Cryo-electron microscopy has revealed that HIV-1 virions carry a mean of 14 Env trimers. ${ }^{40}$ Thus, antibodies must block 12 or more Env trimers on average to prevent HIV-1 entry. These considerations may help define targets for antibody elicitation using vaccination or the administration of such antibodies using passive immunization strategies. The observation that gp41 trimers move to within $\sim 3.5 \mathrm{~nm}$ at the point of lipid contact formation across membranes suggests that preventing such apposition may prevent HIV-1 entry. One can imagine antibodies or other agents that can bind gp41 and occlude a region of $\sim 3.5 \mathrm{~nm}$ around the gp41 on the cell membrane. Such agents may act as novel HIV-1 entry inhibitors. Our study, for the first time, elucidates the important role of the membrane lipidomes in HIV-1 entry. The lipidomes of HIV-1 and host cell membranes comprise many different lipid types. ${ }^{13}$ Simpler membranes that use fewer lipid types did not lead to successful membrane fusion initiation in our simulations. Two major effects allowed biologically realistic membrane lipidomes to facilitate fusion. First, membrane bending resulted in some lipids splaying more, viz. DOPS and DOPS, than the others, viz. DPPC and DPPE, with the former absorbing much of the bending stress and conferring flexibility to the membrane while the latter potentially maintaining membrane integrity. Membranes with fewer lipid types may not achieve this apportioning required to strike a balance between flexibility and integrity, rendering fusion difficult. Second, the lipidomes interacted with gp41 functional domains, which altered local lipid compositions in a way that reduced the repulsive forces between the membranes. Specifically, we found prominent relocalization of cholesterol near gp41, which because of its hydrophobic nature reduced hydration repulsions. Importantly, when multiple gp41 trimers were closely positioned, the effect reached a threshold that allowed lipid contact formation and mixing. Thus, the concerted activity of multiple gp41 trimers and the lipidomes was involved. Interventions could consider altering the lipidomes, which may prevent such lipid reorganization and preclude fusion. Indeed, altering membrane cholesterol has already been shown to compromise HIV-1 fusion. ${ }^{41}$

Our simulations have focused on the narrow window in the HIV-1 entry process between gp41 anchoring and lipid mixing. This window, however, is a crucial one and requires detailed simulations involving both membrane structures, viral structures, lipids, and proteins. The events preceding gp41 
anchoring are extracellular and can be studied without explicit membrane constructions. The events postlipid mixing rely less on the role of proteins and lipidome diversity and thus can be studied using simpler model membranes without proteins. Our study, thus, addresses the most challenging part of the entry process. Indeed, we are thus able to unravel the crucial roles of the lipidomes and the cooperative functions of multiple gp41 trimers. Future studies may integrate our findings into largerscale simulations starting with gp120-CD4 binding and culminating in fusion pore formation, which may soon become tractable as quantum computing technologies mature.

\section{MATERIALS AND METHODS}

Model Building. gp41 Trimer. The modeled structure of the ecto-transmembrane domain of the HIV-1 gp41 postfusion (PoF) six-helix bundled (6HB) conformer was borrowed from our previous work. ${ }^{42}$ During model building we did not use the recent TMD (PDB ID: 5JYN) ${ }^{43}$ and MPER (PDB ID: $6 \mathrm{E} 8 \mathrm{~W})^{44}$ structures as template. ${ }^{38,39}$ In order to assess the quality of our modeled structure with recently available experiments, we have superimposed (Figure S11) the TMD domain of the structure of the gp41 trimer used in the present study with the recent experimental TMD coordinates available (PDB ID: 5JYN). The side and top views of alignment indicate that the overall structure of TMD is similar to the experimental structure, but the helices of 5JYN are relatively more compact than the modeled structure. Notably, the orientation of the conserved Arg696 residue at the center of the TMD, probably important for TMD's stability in the membrane environment and also crucial for viral fusion, ${ }^{45}$ is comparable. To further assess the quality of our modeled gp41 trimer, we have performed Ramachandran plot analysis ${ }^{46}$ which shows that 93.2\% of residues of the modeled structure are in the most favored regions, $4.7 \%$ are in the additional allowed regions, $1.7 \%$ are in the generously allowed regions, and $0.4 \%$ residues are in the disallowed regions. ProSA ${ }^{47}$ shows that the quality of our structure lies within the range of available high-resolution experimental structures (Figure S11). Our model also passed the WHATCHECK's ${ }^{48}$ structure evaluation norms (data not shown). We speculate that the invasion mechanism of HIV-1 to the host cell is only marginally affected by the minor structural difference in our modeled structure.

The invasion mechanism of HIV-1 initially entails the spike proteins, gp120, binding to the host cell receptor/coreceptor. After initial binding, the fusion protein, gp41, perturbs the host cell membrane and further undergoes drastic conformational changes from the prehairpin intermediate conformer (nearly straight) to the postfusion six-helix bundle (6HB) conformer. The conformational transitioning of gp41 drags the HIV-1 and human cell membranes to form a stalk and initiates membrane fusion. ${ }^{49}$ Understanding the conformational transition of the gp41 trimer is a nontrivial problem to be probed using MD simulations studies. Thus, to study the invasion of HIV-1 into a human cell, we have splayed the FP+FPPR and MPER+TMD domains of the $6 \mathrm{HB}$ conformer of the gp41 trimer (Figure $\mathrm{S} 1 \mathrm{~B})$ to bridge the cell membrane models. The coordinates of the splayed conformer are available online (https://zenodo. org/record/4159321\#.X5vT64gzY2w). While connecting the membranes, the fusion peptide (FP) domain of gp41 is inserted into the upper leaflet of the host membrane, and the transmembrane domain (TMD) spans the HIV-1 membrane. The Martini coarse-grained (CG) model of the customized atomistic model of the gp41 trimer was obtained from the CHARMM-GUI web portal. ${ }^{50,51}$

Human and HIV-1 Cell Membranes. A bilayer (dimension $40 \times 40 \mathrm{~nm}^{2}$ ) and a vesicle (diameter $\sim 20 \mathrm{~nm}$ ) composed of mixed CG lipid types mimicking near biological compositions of the human T-cell membrane (DPPC: $13.7 \%$; DPPE: $8.7 \%$; DOPS: 6.7\%; POPC: $15.8 \%$; POPE: $10.5 \%$; POSM: $7.3 \%$; and Chol: $37.2 \%$ ) lipidome ${ }^{13,52}$ were constructed using the Martini builder at the CHARMM-GUI web interface. A vesicle (diameter $\sim 20 \mathrm{~nm}$ ) with near biological lipid composition (POPC: 9.8\%, POPE: 18\%; POPS: 6.4\%; POSM: 20\%; and Chol: $45.7 \%$ ) of the HIV-1 cell membrane ${ }^{13,26,52}$ was generated using the Martini builder at the CHARMM-GUI web interface. The inner and outer layers of cell membrane models were asymmetrically composed of eight different types of lipids with different compositions (Table S4). The HIV-1 vesicle generated by the CHARMM-GUI server contains 6 holes to allow passage of water and flip-flop of lipids between leaflets. The holes were gradually closed during equilibration stages and further simulated for $5 \mu \mathrm{s}$. The stable HIV-1 vesicle after the production run, with limited water, was used for the fusion study in this study. For more details see Figure S12.

Human-gp41-HIV-1 Complex. To study the gp41 mediated fusion mechanism of HIV-1 with the human cell, we bridged the HIV-1 and human cell membrane CG models by gp41 trimers. The minimal distance between the HIV-1 and human cell membranes was maintained $\sim 8 \AA$ initially. The TMD domain spans the HIV-1 membrane, and the FP domain of the gp41 trimer was inserted in the upper leaflet of the host membrane. The lipids of the membranes within $4 \AA$ of the FP and TMD of the gp41 were deleted to remove any bad contacts. We build different human-gp41-HIV-1 complexes, where the count of gp41 trimers varies from zero to four. The ternary complex of the human membrane, the gp41 trimer, and the HIV-1 vesicle was placed in a box with a dimension of $40 \times$ $40 \times 36 \mathrm{~nm}^{3}$. The box with the complex was solvated by standard Martini water beads and $0.14 \mathrm{M} \mathrm{NaCl}$ salt.

Simulation Details. Simulations were performed using Gromacs version 5.1.4 $4^{53}$ with standard Martini v2.2 force field for protein, lipids, water, and ions. ${ }^{54}$ The energy of the systems was initially minimized by the steepest descent algorithm with a force tolerance of $100 \mathrm{~kJ} / \mathrm{mol} / \mathrm{nm}$. Equilibration of the systems was achieved by multiple stages, during which restraints applied on the lipids were gradually released and integration time steps were steadily increased from 10 to $30 \mathrm{fs}$. All the simulations were performed with periodic boundary conditions (PBCs) to simulate bulk behavior. The dielectric constant was set to 15 . The long-range electrostatic Coulombic interactions were truncated with a shift cutoff of $1.2 \mathrm{~nm}$, and the Lennard-Jones ( $\mathrm{LJ})$ potential was smoothly shifted to zero within 0.9 to $1.2 \mathrm{~nm}$ during CGMDS in this study. The pressure was maintained at 1 bar using a Berendsen barostat during equilibration and production simulations. Semiisotropic pressure coupling was adapted for all the systems, except for vesicle systems we applied isotropic coupling. The temperature was adjusted using Berendsen and V-rescale thermostats ${ }^{55}$ during equilibration and production simulations, respectively. The gp41, HIV-1 vesicle, human bilayer, and solvent were coupled separately with a time constant of 1 ps. The neighbor list was updated at every 5 steps during the CGMDS with a 30 fs time step.

CGMDS with Varied Number of gp41 Trimers Anchored. The simulation of solvated systems containing only the HIV-1 
vesicle and human membrane models (vesicle and bilayer), without gp41 trimers, was simulated for $5 \mu \mathrm{s}$ each at $300 \mathrm{~K}$. Further, the systems containing the HIV-1 vesicle, human bilayer, and bridged HIV-1 gp41 trimers (count varies from one to four) were simulated for $10 \mu \mathrm{s}$ each at $300 \mathrm{~K}$.

CGMDS at Varying Temperatures. The simulation of systems containing the vesicle, bilayer, and four HIV-1 gp41 trimers is only selected for stalk energy barrier estimation and was further simulated at five different temperatures, viz. 300, $320,340,360$, and $400 \mathrm{~K}$. Each simulation was performed for 2 $\mu \mathrm{s}$ and repeated twice with different starting velocities.

CGMDS of POPC/POPS Bilayer-Vesicle with Four gp41 Trimers at Varying Temperatures. The simulation of the bilayer and a vesicle composed of POPC (80\%) and POPS (20\%) lipids with four gp41 trimers was performed at 300 and $400 \mathrm{~K}$ for $10 \mu \mathrm{s}$ each. All simulations in the current study are bereft of any external force, electric field, membrane tension, and biased lipid compositions.

Data Analysis. All the analyses were carried out by using in-house tcl codes and/or the tools available in the GROMACS package. Movies and snapshots were made using Visual Molecular Dynamics software. ${ }^{56}$ Any lipid from the human bilayer moving more than $15 \AA$ above from the geometrical center of the FP and TMD domain of four gp41 trimers is considered to be transferred from the human cell membrane to the HIV-1 vesicle $(\mathrm{C} \rightarrow \mathrm{H})$. Similarly, any lipid from the vesicle moving more than $15 \AA$ below from the geometrical center of the FP and TMD domain of four gp41 trimers is considered to be transferred from the HIV-1 vesicle to the human cell membrane $(\mathrm{H} \rightarrow \mathrm{C})$.

\section{ASSOCIATED CONTENT}

\section{(s) Supporting Information}

The Supporting Information is available free of charge at https://pubs.acs.org/doi/10.1021/acs.jcim.0c01291.

Figure S1, functional domains and splayed conformer of HIV-1 gp41; Figure S2, simulation of membrane models in absence of gp41; Figure S3, simulation of human and HIV-1 membrane models in presence of gp 41; Figure S4, extension of simulation of membrane models in presence of two gp41 trimers; Figure S5, simulation of human and HIV-1 membrane models with arbitrary lipid compositions; Figure S6, lipid tilt distribution map; Figure S7, morphology of human cell membrane during stalk initiation; Figure S8, histogram plot of lipid fractions; Figure S9, tilt angle of gp41 functional domains; Figure S10, stalk initiation snapshot; Figure S11, structural superimposition and quality assessment of modeled structure; Figure S12, equilibration of HIV-1 vesicle; Table S1, lateral diffusion of lipids; Table S2, error in initiation time at each simulation temperature; Table S3, details of contact time and $\Delta G_{S}$; and Table S4, lipid composition of HIV-1 cell membrane and human T-cell membrane (PDF)

Movie S1, human-HIV-1 intermembrane lipid transfer (MP4)

\section{AUTHOR INFORMATION}

\section{Corresponding Author}

Prabal K. Maiti - Center for Condensed Matter Theory,

Department of Physics, Indian Institute of Science, Bangalore
560012, India; ㅇo이.org/0000-0002-9956-1136;

Email: maiti@iisc.ac.in

\section{Authors}

Biswajit Gorai - Center for Condensed Matter Theory, Department of Physics, Indian Institute of Science, Bangalore 560012, India; orcid.org/0000-0003-1001-5567

Anil Kumar Sahoo - Center for Condensed Matter Theory, Department of Physics, Indian Institute of Science, Bangalore 560012, India; (1) orcid.org/0000-0001-7769-4774

Anand Srivastava - Molecular Biophysics Unit, Biological Sciences Division, Indian Institute of Science, Bangalore 560012, India; 이이.org/0000-0002-2757-1511

Narendra M. Dixit - Department of Chemical Engineering and Centre for Biosystems Science and Engineering, Indian Institute of Science, Bangalore 560012, India; 이이.org/ 0000-0002-2145-9828

Complete contact information is available at:

https://pubs.acs.org/10.1021/acs.jcim.0c01291

\section{Author Contributions}

B.G. and P.K.M. designed the research; B.G. performed the research; A.K.S., B.G., and N.M.D. analyzed the data; B.G. wrote the first draft with help from A.K.S. and P.K.M. All authors reviewed the simulation data, analyses, and figures. All authors cowrote the final manuscript.

\section{Notes}

The authors declare no competing financial interest.

\section{ACKNOWLEDGMENTS}

B.G. acknowledges the Dr. D. S. Kothari Post-Doctoral Fellowship (DSKPDF), UGC, India for financial support [201718-BL/16-17/0437]. We acknowledge Sahasrat, SERC, and TUE-CMS, SSCU at IISc, Bangalore, India for the computational facilities.

\section{REFERENCES}

(1) Blumenthal, R.; Durell, S.; Viard, M. HIV entry and envelope glycoprotein-mediated fusion. J. Biol. Chem. 2012, 287, 40841-40849.

(2) Wilen, C. B.; Tilton, J. C.; Doms, R. W. HIV: cell binding and entry. Cold Spring Harbor Perspect. Med. 2012, 2, a006866.

(3) Ward, A. B.; Wilson, I. A. Insights into the trimeric HIV-1 envelope glycoprotein structure. Trends Biochem. Sci. 2015, 40, 101107.

(4) Liu, J.; Bartesaghi, A.; Borgnia, M. J.; Sapiro, G.; Subramaniam, S. Molecular architecture of native HIV-1 gp120 trimers. Nature 2008, 455, 109.

(5) Dorr, P.; Westby, M.; Dobbs, S.; Griffin, P.; Irvine, B.; Macartney, M.; Mori, J.; Rickett, G.; Smith-Burchnell, C.; Napier, C.; Webster, R.; Armour, D.; Price, D.; Stammen, B.; Wood, A.; Perros, M. Maraviroc (uk-427,857), a potent, orally bioavailable, and selective small-molecule inhibitor of chemokine receptor CCR5 with broadspectrum anti-human immune deficiency virus type 1 activity. Antimicrob. Agents Chemother. 2005, 49, 4721-4732.

(6) Nandy, B.; Saurabh, S.; Sahoo, A. K.; Dixit, N. M.; Maiti, P. K. The SPL7013 dendrimer destabilizes the HIV-1 gp120-CD4 complex. Nanoscale 2015, 7, 18628-18641.

(7) Nandy, B.; Bindu, D. H.; Dixit, N. M.; Maiti, P. K. Simulations reveal that the HIV-1 gp120-CD4 complex dissociates via complex pathways and is a potential target of the polyamidoamine (PAMAM) dendrimer. J. Chem. Phys. 2013, 139, 024905.

(8) Kilby, J. M.; Hopkins, S.; Venetta, T. M.; DiMassimo, B.; Cloud, G. A.; Lee, J. Y.; Alldredge, L.; Hunter, E.; Lambert, D.; Bolognesi, D.; Matthews, T.; Johnson, M. R.; Nowak, M. A.; Shaw, G. M.; Saag, M. S. Potent suppression of HIV-1 replication in humans by T-20, a 
peptide inhibitor of gp41-mediated virus entry. Nat. Med. 1998, 4, 1302-1307.

(9) Burton, D. R.; Hangartner, L. Broadly neutralizing antibodies to HIV and their role in vaccine design. Annu. Rev. Immunol. 2016, 34, 635-659.

(10) Brandenberg, O. F.; Magnus, C.; Rusert, P.; Regoes, R. R.; Trkola, A. Different infectivity of HIV-1 strains is linked to number of envelope trimers required for entry. PLoS Pathog. 2015, 11, No. e1004595.

(11) McDargh, Z. A.; Polley, A.; O'Shaughnessy, B. SNAREmediated membrane fusion is a two-stage process driven by entropic forces. Biophys. J. 2019, 116, No. 314a.

(12) Harrison, S. C. Mechanism of membrane fusion by viral envelope proteins. Adv. Virus Res. 2005, 64, 231-261.

(13) Brügger, B.; Glass, B.; Haberkant, P.; Leibrecht, I.; Wieland, F. T.; Kräusslich, H. G. The HIV lipidome: a raft with an unusual composition. Proc. Natl. Acad. Sci. U. S. A. 2006, 103, 2641-2646.

(14) Knecht, V.; Marrink, S. J. Molecular dynamics simulations of lipid vesicle fusion in atomic detail. Biophys. J. 2007, 92, 4254-4261.

(15) Shillcock, J. C.; Lipowsky, R. Tension-induced fusion of bilayer membranes and vesicles. Nat. Mater. 2005, 4, 225.

(16) Marrink, S. J.; Mark, A. E. The mechanism of vesicle fusion as revealed by molecular dynamics simulations. J. Am. Chem. Soc. 2003, $125,11144-11145$.

(17) Semrau, S.; Schmidt, T. Membrane heterogeneity-from lipid domains to curvature effects. Soft Matter 2009, 5, 3174-3186.

(18) Yang, S. T.; Kiessling, V.; Simmons, J. A.; White, J. M.; Tamm, L. K. HIV gp41-mediated membrane fusion occurs at edges of cholesterol-rich lipid domains. Nat. Chem. Biol. 2015, 11, 424.

(19) Shinoda, W.; Klein, M. Effective interaction between small unilamellar vesicles as probed by coarse-grained molecular dynamics simulations. Pure Appl. Chem. 2014, 86, 215-222.

(20) Schneck, E.; Sedlmeier, F.; Netz, R. R. Hydration repulsion between biomembranes results from an interplay of dehydration and depolarization. Proc. Natl. Acad. Sci. U. S. A. 2012, 109, 14405-14409.

(21) Chojnacki, J.; Waithe, D.; Carravilla, P.; Huarte, N.; Galiani, S.; Enderlein, J.; Eggeling, C. Envelope glycoprotein mobility on HIV-1 particles depends on the virus maturation state. Nat. Commun. 2017, 8,545 .

(22) Enkavi, G.; Javanainen, M.; Kulig, W.; Róg, T.; Vattulainen, I. Multiscale simulations of biological membranes: The challenge to understand biological phenomena in a living substance. Chem. Rev. 2019, 119, 5607-5774.

(23) Smirnova, Y. G.; Marrink, S. J.; Lipowsky, R.; Knecht, V. Solvent-exposed tails as prestalk transition states for membrane fusion at low hydration. J. Am. Chem. Soc. 2010, 132, 6710-6718.

(24) Kawamoto, S.; Klein, M. L.; Shinoda, W. Coarse-grained molecular dynamics study of membrane fusion: Curvature effects on free energy barriers along the stalk mechanism. J. Chem. Phys. 2015, 143, 243112.

(25) Wang, W.; Yang, L.; Huang, H. Evidence of cholesterol accumulated in high curvature regions: Implication to the curvature elastic energy for lipid mixtures. Biophys. J. 2007, 92, 2819-2830.

(26) Aloia, R. C.; Tian, H.; Jensen, F. C. Lipid composition and fluidity of the human immunodeficiency virus envelope and host cell plasma membranes. Proc. Natl. Acad. Sci. U. S. A. 1993, 90, 51815185.

(27) Barenholz, Y.; Moore, N. F.; Wagner, R. R. Enveloped viruses as model membrane systems: microviscosity of vesicular stomatitis virus and host cell membranes. Biochemistry 1976, 15, 3563-3570.

(28) Apajalahti, T.; Niemelä, P.; Govindan, P. N.; Miettinen, M. S.; Salonen, E.; Marrink, S. J.; Vattulainen, I. Concerted diffusion of lipids in raft-like membranes. Faraday Discuss. 2010, 144, 411-430.

(29) Niemelä, P. S.; Ollila, S.; Hyvönen, M. T.; Karttunen, M.; Vattulainen, I. Assessing the nature of lipid raft membranes. PLoS Comput. Biol. 2007, 3, No. e34.

(30) Marrink, S. J.; De Vries, A. H.; Mark, A. E. Coarse grained model for semiquantitative lipid simulations. J. Phys. Chem. B 2004, $108,750-760$.
(31) Cai, L.; Gochin, M.; Liu, K. Biochemistry and biophysics of HIV-1 gp41-membrane interactions and implications for HIV-1 envelope protein mediated viral-cell fusion and fusion inhibitor design. Curr. Top. Med. Chem. 2011, 11, 2959-2984.

(32) Vishwanathan, S. A.; Thomas, A.; Brasseur, R.; Epand, R. F.; Hunter, E.; Epand, R. M. Hydrophobic substitutions in the first residue of the CRAC segment of the gp41 protein of HIV. Biochemistry 2008, 47, 124-130.

(33) Melikyan, G. Membrane fusion mediated by human immunodeficiency virus envelope glycoprotein. Curr. Top. Membr. 2011, 68, 81-106.

(34) Kozlovsky, Y.; Kozlov, M. M. Stalk model of membrane fusion: solution of energy crisis. Biophys. J. 2002, 82, 882-895.

(35) Brandenberg, O.; Magnus, C.; Regoes, R.; Trkola, A. The HIV1 entry process: A stoichiometric view. Trends Microbiol. 2015, 23, $763-774$.

(36) Sougrat, R.; Bartesaghi, A.; Lifson, J. D.; Bennett, A. E.; Bess, J. W.; Zabransky, D. J.; Subramaniam, S. Electron tomography of the contact between $\mathrm{T}$ cells and SIV/HIV-1: implications for viral entry. PLoS Pathog. 2007, 3, e63.

(37) Zhang, Y.; Dudko, O. K. Statistical mechanics of viral entry. Phys. Rev. Lett. 2015, 114, 018104.

(38) Huang, J.; Ofek, G.; Laub, L.; Louder, M. K.; Doria-Rose, N. A.; Longo, N. S.; Imamichi, H.; Bailer, R. T.; Chakrabarti, B.; Sharma, S. K.; Alam, S. M.; Wang, T.; Yang, Y.; Zhang, B.; Migueles, S. A.; Wyatt, R.; Haynes, B. F.; Kwong, P. D.; Mascola, J. R.; Connors, M. Broad and potent neutralization of HIV-1 by a gp41-specific human antibody. Nature 2012, 491, 406-412.

(39) Rantalainen, K.; Berndsen, Z. T.; Antanasijevic, A.; Schiffner, T.; Zhang, X.; Lee, W. H.; Torres, J. L.; Zhang, L.; Irimia, A.; Copps, J.; Zhou, K. H.; Kwon, Y. D.; Law, W. H.; Schramm, C. A.; Verardi, R.; Krebs, S. J.; Kwong, P. D.; Doria-Rose, N. A.; Wilson, I. A.; Zwick, M. B.; Yates, J. R., III; Schief, W. R.; Ward, A. B. HIV-1 Envelope and MPER antibody structures in lipid assemblies. Cell Rep. 2020, 31, 107583.

(40) Zhu, P.; Liu, J.; Bess, J.; Chertova, E.; Lifson, J. D.; Grisé, H.; Ofek, G. A.; Taylor, K. A.; Roux, K. H. Distribution and threedimensional structure of AIDS virus envelope spikes. Nature 2006, $441,847-852$.

(41) Liao, Z.; Graham, D. R.; Hildreth, J. E. Lipid rafts and HIV pathogenesis: virion-associated cholesterol is required for fusion and infection of susceptible cells. AIDS Res. Hum. Retroviruses 2003, 19, 675-687.

(42) Gorai, B.; Das, S.; Maiti, P. K. Prediction and validation of HIV-1 gp41 ecto-transmembrane domain post-fusion trimeric structure using molecular modeling. J. Biomol. Struct. Dyn. 2020, 38, 2592-2603.

(43) Dev, J.; Park, D.; Fu, Q.; Chen, J.; Ha, H. J.; Ghantous, F.; Herrmann, T.; Chang, W.; Liu, Z.; Frey, G.; Seaman, M. S.; Chen, B.; Chou, J. J. Structural basis for membrane anchoring of HIV-1 envelope spike. Science 2016, 353, 172-175.

(44) Fu, Q.; Shaik, M. M.; Cai, Y.; Ghantous, F.; Piai, A.; Peng, H.; Rits-Volloch, S.; Liu, Z.; Harrison, S. C.; Seaman, M. S.; Chen, B.; Chou, J. J. Structure of the membrane proximal external region of HIV-1 envelope glycoprotein. Proc. Natl. Acad. Sci. U. S. A. 2018, 115, E8892-8899.

(45) Hollingsworth IV, L. R.; Lemkul, J. A.; Bevan, D. R.; Brown, A. M. HIV-1 Env gp41 transmembrane domain dynamics are modulated by lipid, water, and ion interactions. Biophys. J. 2018, 115, 84-94.

(46) Ramachandran, G. N.; Ramakrishnan, C.; Sasisekharan, V. Stereochemistry of polypeptide chain configurations. J. Mol. Biol. 1963, 7, 95-99.

(47) Wiederstein, M.; Sippl, M. J. ProSA-web: interactive web service for the recognition of errors in three-dimensional structures of proteins. Nucleic Acids Res. 2007, 35 (suppl_2), W407-W410.

(48) Vriend, G.; Sander, C. Quality control of protein models: directional atomic contact analysis. J. Appl. Crystallogr. 1993, 26, 4760. 
(49) Chen, B. Molecular mechanism of HIV-1 entry. Trends Microbiol. 2019, 27, 878-891.

(50) Jo, S.; Kim, T.; Iyer, V. G.; Im, W. CHARMM-GUI: a webbased graphical user interface for CHARMM. J. Comput. Chem. 2008, 29, 1859-1865.

(51) Qi, Y.; Ingólfsson, H. I.; Cheng, X.; Lee, J.; Marrink, S. J.; Im, W. CHARMM-GUI Martini Maker for coarse-grained simulations with the Martini force field. J. Chem. Theory Comput. 2015, 11, 44864494.

(52) Aloia, R. C.; Jensen, F. C.; Curtain, C. C.; Mobley, P. W.; Gordon, L. M. Lipid composition and fluidity of the human immunodeficiency virus. Proc. Natl. Acad. Sci. U. S. A. 1988, 85, 900-904.

(53) Berendsen, H. J.; van der Spoel, D.; van Drunen, R. Gromacs: a message-passing parallel molecular dynamics implementation. Comput. Phys. Commun. 1995, 91, 43-56.

(54) De Jong, D.; Singh, G.; Bennett, W. D.; Arnarez, C.; Wassenaar, T. A.; Scháfer, L. V.; Periole, X.; Tieleman, D. P.; Marrink, S. J. Improved parameters for the Martini coarse-grained protein force field. J. Chem. Theory Comput. 2013, 9, 687-697.

(55) Berendsen, H. J.; Postma, J. V.; van Gunsteren, W. F.; DiNola, A. R.; Haak, J. R. Molecular dynamics with coupling to an external bath. J. Chem. Phys. 1984, 81, 3684-3690.

(56) Humphrey, W.; Dalke, A.; Schulten, K. VMD: visual molecular dynamics. J. Mol. Graphics 1996, 14, 33-38. 